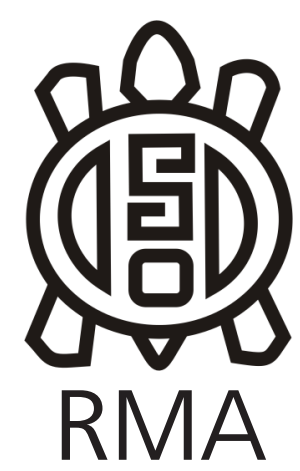

\title{
Límites del culto estatal y alcances del culto popular en el-Amarna (Egipto)
}

\author{
Boundaries of the state religion and extension of the popular religion \\ at el-Amarna (Egypt) \\ María Laura lamarino*
}

Arqueología

\section{Resumen}

Este artículo busca revisar algunos postulados acerca de la reforma planteada durante el reinado de Akhenatón (1355-1338 A.C.) y las características del uso de los espacios de lo que fue el centro religioso y político emplazado en el-Amarna. Se actualizan los datos acerca de la ocupación y duración del período de Amarna mediante la revisión del uso de los espacios planeados y no planeados, asociados con evidencia material de prácticas religiosas, tanto oficiales (ligadas con el culto del Atón) como extraoficiales (asociadas con la religión tradicional). Así, se someterán los datos a pruebas estadísticas con el fin de analizar la distribución de los objetos relacionados con el ámbito religioso para determinar el alcance de los distintos tipos de cultos que convivieron en los espacios de Amarna.

Palabras clave: Amarna; reforma; práctica religiosa; religión no estatal; Atón.

\begin{abstract}
The aim of this paper is to review several assumptions about the reform proposed during the reign of Akhenaten (1355-1338 B.C.) and the characteristics of the use of the spaces in el-Amarna. We will update the data on the occupation and duration of the Amarna period through a review of the use of planned and unplanned spaces associated with material evidence of religious practices, both official (linked to the cult of the Aten) and unofficial (related to traditional religion). Thus, we will use statistical testing to analyze the distribution of objects related to the religious sphere to determine the scope of the diversity of cults that coexisted in the Amarna areas.
\end{abstract}

Keywords: Amarna; reform; religious practice; non state religion; Aten.

\section{Introducción}

El uso y ocupación de los espacios en la antigüedad egipcia incluyó la intervención de agentes estatales y no estatales. En el contexto de la reforma llevada a cabo por el faraón Akhenatón (Amenhotep IV) (1355-1338 A.C.), el asentamiento el-Amarna (o Amarna), no fue una excepción, ya que se lo planeó para que la familia real desarrollara el culto al disco solar o Atón (itn). Durante el período de Amarna, esta deidad fue exaltada por el estado en un plano de exclusividad, lo cual llevó a que varios autores lo identifiquen con una etapa monoteista de la historia egipcia (Redford 1984; Aldred 1988; Assmann 1989; Hornung 1999 [1971]). La evidencia aquí presentada invita a relativizar el empleo de ese término como rector en la dinámica de uso del sitio y a integrar en el análisis la intervención de agentes no estatales'.

Las primeras publicaciones de los materiales recuperados

\footnotetext{
${ }^{1}$ Las ideas presentadas en este trabajo son resultado de la revisión y ampliación de las expuestas en una versión preliminar en la V SEMNA - Semana de Egiptologia do Museu Nacional (Brasil, Rio de Janeiro).
}

mediante excavaciones realizadas en el sitio arqueológico Amarna fueron llevadas a cabo por Flinders Petrie, el Deutsche Orient-Gesellschaft (DOG) y la Egiptian Exploration Society (EES) (Stevens 2015). A partir de ellas fue posible plantear la existencia de una práctica religiosa compleja en la antigua Akhetatón, la cual exedió el culto al Atón y a la familia real. Los análisis iniciales de los hallazgos de Petrie a fines del siglo XIX llevaron a catalogar más de 100 relacionados con cultos no atonianos encontrados en distintas áreas (Petrie 1894: pl. XV-XVII). Luego, las sucesivas campañas de la EES permitieron reconocer inscripciones y otros materiales conectados con el culto a dedidades tradicionales y/o populares, e incluso Peet en coautoría con Woolley (1923: 66 ; 95) dejaron constancia de evidencia asociada con Amón. Si bien en estos trabajos constaba la adoración de deidades tradicionales en el centro dedicado al Atón, no profundizaban en cuándo, cómo y por quién esos dioses fueron adorados. A partir de las excavaciones dirigidas por Barry Kemp, iniciadas en 1977, se incluyeron nuevos hallazgos, permitiendo continuar las interpretaciones sobre la práctica religiosa desarrollada en Amarna (Shaw 
1984; Kemp 1995b, 2012; Stevens 2003, 2006, 2009, 2011, 2012; Bickel 2003, entre otros).

Con el objetivo de profundizar en el reconocimiento de las características de las diferentes áreas de el-Amarna y cómo estas fueron afectadas por las prácticas religiosas desarrolladas en ellas, se analizará la relación entre la cantidad y la distribución de evidencia material asociada a la religión en el sitio. Se considerarán las distintas expresiones de la religiosidad para el período, a saber: la patrocinada por el estado -y centrada en Atón y la familia real- y la que se desarrolló por fuera de ese patrocinio -ligada a las deidades tradicionales-.

El recorte temporal que abarca este trabajo requiere establecer cuándo fueron adoradas las deidades tradicionales en Amarna. Para ello se deben determinar las características y extensión en el tiempo de ocupación de cada área, ya que las mismas no habrían sido desocupadas al mismo tiempo. El período de ocupación de Akhetatón como residencia real fue de entre 16 y 17 años (Kemp 2012). Su fundación está registrada en las estelas fronterizas (Murnane y van Siclen 1993) pero la fecha exacta de su abandono no es tan clara. Durante los reinados de los sucesores de Akhenatón se produjo la restauración religiosa a las formas previas a la reforma, pero también se mantuvo el uso de Amarna hasta el desmantelamiento de sus edificios más importantes y administrativamente nodales, que luego fueron reutilizados en otras construcciones de todo Egipto (Hoffmeier 2015).

Respecto del momento en el que se desarrollaron los cultos tradicionales, los hallazgos asociados los ubican dentro del contexto de la restauración de Tutankhamón. Sin embargo, esto se debe revisar si se considera que la evidencia material proveniente de Amarna, como la inscripción del nombre previo de aquel faraón (Tutankhatón), en la que la relevancia de la estructura nominativa da cuenta de la adhesión al culto de Atón.

Además, otros materiales depositados en la tumba de Tutankamón evocan estilos y temáticas típicas del período de Amarna (Silverman et al. 2006: 165) que habilitan plantear un breve tiempo en el que, aún desaparecido Akhenatón, la restauración todavía no era un hecho en el sitio (Kemp 2012: 301). Por ello, si la imposición de la restauración, así como la reforma, se consideran procesos graduales, y no abruptos, los cultos tradicionales pudieron aparecer tanto en el período de un faraón como de otro, sin ser la sucesión regia, indicador de automática restauración religiosa para toda la población que habitaba Amarna. En este sentido, existe la posibilidad de que la adoración a otros dioses haya sido contemporánea al Atón.

La manera en que los cultos tradicionales fueron practicados en Amarna, requiere detallar aspectos en relación con quiénes practicaban estos cultos, para qué lo hacían, dónde se desarrollaban y cuál era el grado de importancia que representaban en la vida religiosa del centro atoniano.

\section{El- Amarna: la ciudad y la reforma}

El traslado del centro político y religioso desde Tebas a Akhetatón (o 3ht itn, 'Horizonte de Atón') consistió en la elección de un sector geográfico específico donde se construyó un nuevo asentamiento polifuncional $\left(\mathrm{O}^{\prime}\right.$ Connor 1989) que preveía una planificación territorial acorde con el reemplazo del culto estatal del dios Amón (y su poderosa elite) por el de Atón, posicionando a este último como el más importante de Egipto.

Según la esquematización ofrecida por Kemp (1992 [1989]) Amarna se divide en áreas diferenciables por sus características, ubicación territorial o función (Figura 1). La Ciudad Norte estaba en el límite del asentamiento y se consideraba la residencia del faraón, luego el Palacio Norte, destinado a actividades administrativas y/o diplomáticas (Kemp 2012) y la zona residencial del Suburbio Norte. En el área de la Ciudad Central se situaron los principales palacios, templos al Atón y edificios administrativos, además era el punto de llegada de la 'vía real' por la cual desfilaban el faraón y su familia desde el Palacio Norte. Al Sur se emplazaba una zona de residencias conocida como la Ciudad Principal, luego el residencial Suburbio Sur, el templo de Kom el-Nana y, todavía más al Sur, el templo solar Marú Atón. Al Sureste de la Ciudad Central, en el desierto, se ubicaba una aldea amurallada denominada Pueblo de Trabajadores donde habitaron los artesanos que se desempeñaron en las tumbas de la elite y del faraón y la familia real ${ }^{2}$. Entre el ingreso al wadi real y las tumbas Sur se encontraba el Pueblo de Piedra, área residencial más pequeña que el Pueblo de Trabajadores, y que habría alojado una comunidad conectada con la construcción (Stevens 2012).

Las áreas relacionadas directamente con actividades oficiales -templos, palacios, edificios administrativos y de producción estatal y residencias de elite- fueron planeadas y construidas por el mismo estado mientras que, en paralelo al plan original, se asentaba el resto de la población. Asimismo, el patrón de asentamiento de los habitantes de Amarna debió ser influido por el corto tiempo en el que se construyó, así como también por el hecho de que se requirió del traspaso de población de las distintas regiones de Egipto y del extranjero (Kemp 2012: 74).

Los habitantes relacionados directamente con el estado poseían casas de mayor tamaño alrededor de las cuales se agrupaban otras menores (Kemp 2016: 7), en general

\footnotetext{
${ }^{2}$ La comunidad de trabajadores de el-Amarna pudo ser la misma que habitó Deir el-Medina (Kemp 1987: 44-49).
} 


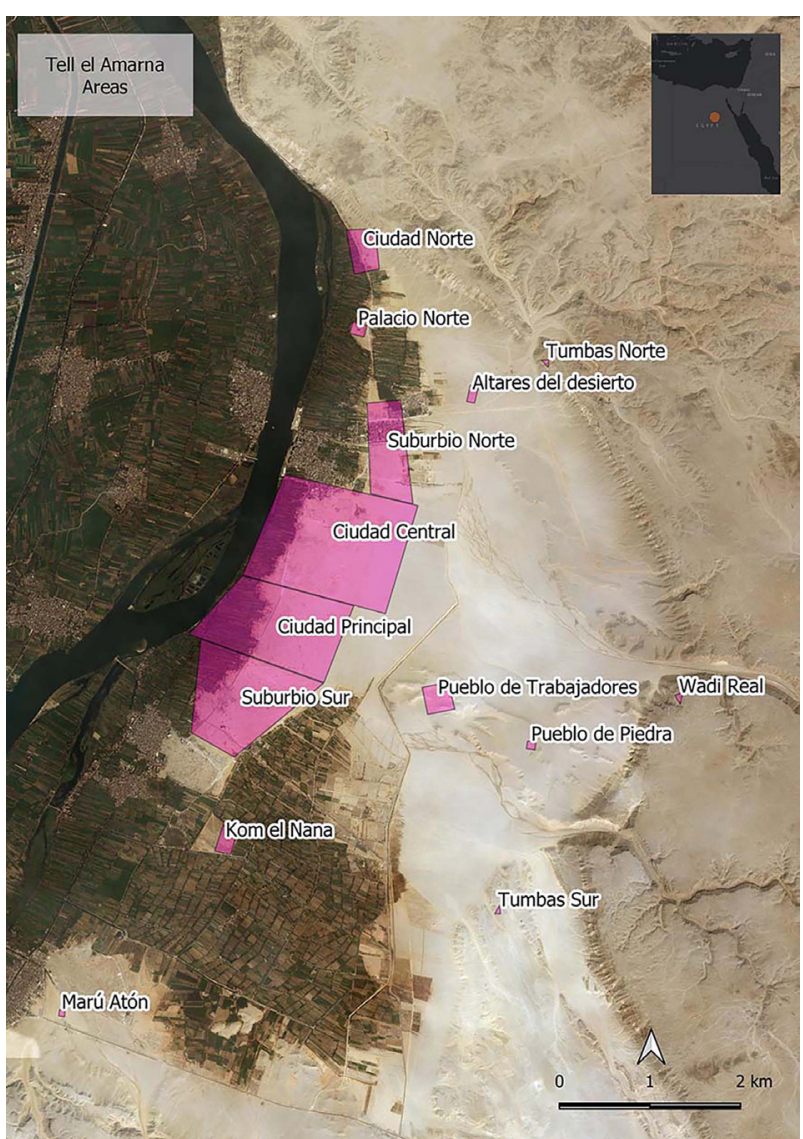

Figura 1. Amarna con división por áreas (Fuente: Google Pro Earth)

Figure 1. Amarna with areas division (Font: Google Pro Earth).

habitadas por quienes dependían de los funcionarios estatales. Estas características habitacionales conforman Amarna como una sumatoria de aldeas donde el tamaño de las casas oficiaba como indicador para determinar si sus habitantes eran más o menos poderosos (Kemp 1992 [1989]: 378) o estaban más directa o indirectamente ligados al estado ${ }^{3}$. En el interior de estas áreas residenciales convivían zonas creadas especialmente para la práctica religiosa tanto atoniana como tradicional o popular.

\section{La reforma de Amarna y el culto estatal}

El traslado a Amarna fue el corolario de una reforma que estaba ya en proceso años antes y se enmarcó en la solarización impulsada por los gobernantes de la dinastía XVIII, principalmente por el predecesor de Akhenatón, Amenhotep III (Johnson 1996). Así, el período de Amarna debe considerarse en el mediano plazo (1393-1298 A.C.) y no asociarse solamente con el tiempo que estuvo habitado el centro político y religioso que le dio nombre.

A partir del establecimiento de la realeza y la elite en el nuevo centro religioso, la conexión con Atón pasó a ser privilegio del faraón y de su familia, por ser estos

\footnotetext{
${ }^{3}$ Otra manera de relevar información al respecto consiste en el estudio de las tumbas de los funcionarios de Amarna, ya que arrojan evidencias acerca de su vinculación con el aparato estatal (Yomaha 2017).
}

los ejecutantes del culto y únicos intermediarios con la deidad. Mientras que desde el Reino Antiguo el dios sol Ra oficiaba como la síntesis del resto de los dioses que participaban en la creación egipcia, durante el período de Akhenatón, Atón excluyó al resto de los dioses en la creación ${ }^{4}$. Según plantea Hornung (1999 [1971]), cuando se hizo excluyente e intolerante con respecto a los otros dioses, el epíteto de 'único', atribuible a los dioses, tomó el significado que actualmente se relaciona con él, en el sentido literal de exclusividad.

Además de los templos de la Ciudad Central, se considera que otros espacios como palacios, áreas residenciales y distintos sectores alejados del centro fueron sede de prácticas atonianas (Ikram 1989; Kemp 1995a; Stevens 2006; David 2016; Williamson 2017). Según Kemp (2012: 253) los Altares del Desierto estaban destinados a rendir culto al Atón, con el agregado de que desde su emplazamiento se tenía visión directa de las Tumbas Norte, reforzando la simbología de la estructura ritual de la ciudad (O`Connor 1989). Esto, en línea con la idea de que la ciudad entera era un gran templo a cielo abierto, ya que su diseño, ubicación y relación de sus edificios con respecto al entorno natural, generaron un espacio propicio para la adoración continua al Atón (Kemp, 2012; Mallinson 1999; Manzi et al. 2015).

En cuanto a la participación de las personas en el culto a Atón, se observan posturas diferenciadas. Gunn (1916: 93) planteaba que la religión impulsada por Akhenatón no fue un movimiento que incluyó la esfera popular, sino el producto de algo aprendido por un círculo muy pequeño ligado a la corte ${ }^{5}$. Según Teeter (2011: 187) la relación de Atón con los humanos era nula y, mientras que con los cultos tradicionales se podía llegar a conocer a los dioses y establecer algún vínculo con ellos, en la teología de Amarna se afirmaba que nadie podía acceder al conocimiento sobre el dios. Este aspecto se hace visible mediante inscripciones de la época, como el Himno de Atón, cuya versión inscripta en la tumba de Ay señala que la posibilidad de vida de la humanidad radica en la intermediación del monarca ya que "nadie lo conoce (al Atón), excepto tu hijo (el faraón)" (Murnane y Meltzer 1995: 115). Además, en varias escenas, como la de entrega de recompensa a los funcionarios en la Ventana de Aparición del palacio real, se muestran los rayos del dios sol con manos portando signos de vida y poder apuntando a las narices y bocas de Akhenatón y su familia, pero nunca sobre otras personas (Yomaha 2005). La imposibilidad de todo ser humano ajeno a la familia real de conocer y tener relación con la deidad estatal

\footnotetext{
${ }^{4}$ Estas características llevaron a trazar múltiples teorías sobre el culto al Atón, tildándolo de monoteísmo, henoteísmo, sincretismo, entre otros (Hornung (1999 [1971]: 225).

${ }^{5}$ La decoración de las tumbas privadas de Amarna (Yomaha 2005) da cuenta del denotado esfuerzo por resaltar que la elite se formaba por 'hombres nuevos' (Redford 1984) sin vínculos previos con la tradición amoniana y carentes de lazos parentales.
} 
explica la existencia de altares domésticos dedicados a Akhenatón, Nefertiti y sus hijas; únicos capaces de llegar a la deidad y mediadores entre quien requiriera ayuda y el Atón (Ikram 1989: 101).

Por su parte, Stevens (2015) revisa la necesidad de intermediación de la realeza, siendo que el dios adorado era el mismo disco solar y por lo tanto era visible para todos los seres humanos solo mirando al cielo. Al respecto, Bickel (2003: 26) marca la diferencia entre ver, reconocer o saber del dios, ya que era visible pero no por ello reconocible, y solo el faraón estaba conectado con él por el conocimiento. Esta autora tambien señala que en Amarna la religiosidad individual podía expresarse y la gente podía acceder verbal, visual y emocionalmente al Atón (Bickel 2003: 44). Esto se relaciona con la posible existencia de santuarios al aire libre en terrazas aunque los techos están perdidos y solo disponemos de representaciones de los mismos (Spence 2004: 145), por lo que ese aspecto de la religión no es posible de abordar (Stevens 2015: 82).

En cuanto a la elite de Amarna y sus conexiones con la deidad estatal, sabemos por inscripciones y representaciones en sus tumbas, que accedían al Atón por medio de su relación de lealtad con el faraón (Hornung 1999 [1995]): 56). Sin embargo, los diferentes grados de jerarquía y cercanía con el poder central pudieron determinar diferentes modalidades de acceso al dios (Bickel 2003: 34), obligando a una buena parte de la elite a buscar sus propios lazos con el Atón. Al respecto, Baines (2001) da cuenta de evidencia documental, específicamente cartas, en la que se expresan deseos de bienestar invocando directamente al Atón, preguntas sobre si el receptor de la carta está bien, y una declaración de que el protagonista recurre diariamente al dios para asegurar el bienestar del receptor (Baines 2001: 8). Todo señala que estas invocaciones se podían hacer en nombre de los que estaban ausentes, como una acción individual no mediada.

En capillas del Pueblo de Trabajadores aparecen fragmentos de posibles pinturas murales de la familia real (Kemp 1992 [1989]: 304) y en residencias cercanas a los templos principales se encontró evidencia de cartuchos reales y otros objetos para la adoración a la familia real (Kemp 2012: 244). En la casa del funcionario Panehesy, en la Ciudad Central, una estela de Amenhotep III y Tiy sentados bajo los rayos del sol plantea la adoración a la realeza en su papel de proveedores, práctica que precedía al reinado de Akhenatón y estaba relacionada con la edificación de su predecesor (Stevens 2006: 319).

Luego de la muerte de Akhenatón, los cultos dedicados a Atón y a la familia real sobrevivieron en Amarna, aunque resulta dificil precisar su alcance. Si bien la sucesión fue compleja (Gabolde 1998), mediante referencias a Smenkhakare y a Tutankhamón encontradas en la ciudad
(Silverman et al. 2006) se infiere que Amarna continuó activa. El tipo de evidencia y el estrato de los hallazgos encontrados en el Pueblo de Trabajadores atestigua toda una nueva fase de ocupación que comenzó después de que Tutankhamón llegara al trono (Kemp 1984: 4).

La construcción y ocupación de nuevos edificios después de Akhenatón supone que Amarna continuó creciendo (Shaw 1984: 127). En el Pueblo de Trabajadores se halló evidencia material relacionada con el culto no atoniano que pudo ser parte de una práctica paralela a la propuesta por el estado, aunque también puede ser evidencia de la reinstalación de cultos tradicionales. Los hallazgos de las capillas 525 y 529, que han permitido demostrar que las deidades tradicionales se adoraban en paralelo al Atón, fueron señalados por Peet y Woolley (1923: 95-98) para corroborar la ocupación de Amarna en fecha posterior a la muerte de Akhenatón; aunque, según ellos, sus habitantes eran conscientes de que las ideas del faraón se estaban diluyendo y la vuelta a la ortodoxia era inminente. Sin embargo, la calidad de la estructura y decoración de la capilla principal representa una importante inversión de tiempo y esfuerzo, producto del trabajo de una comunidad con confianza en su permanencia en el sitio (Kemp 1984: 11). La ocupación posterior a Akhenatón impulsa a revisar las características de la continuidad en el uso de este centro, así como la posibilidad de supervivencia del culto atoniano o, por lo menos, un final menos abrupto.

\section{La religión popular en Amarna}

La definición de religión popular para el Egipto antiguo remite a creencias y prácticas llevadas a cabo de manera individual o comunitaria por los egipcios comunes en la vida diaria (Pinch 1993: 325). En tanto, los 'egipcios comunes' son definidos por varios autores como los habitantes no ligados directamente al estado, es decir que no ostentaban un cargo de elite (Teeter 1997: 39; Blyth 2006: 159). Sin embargo, y por las características del asentamiento que aquí se trata, la mayoría de sus habitantes eran dependientes del estado y existían pocas familias fuera de esa estructura (Spence 2010: 297). A su vez, las prácticas de la religión popular, profesada por todos los miembros de la sociedad, no necesariamente se desarrollaron de manera privada, individual o en el interior de los hogares (Friedman 1994: 96). Además, existen diferencias entre práctica religiosa y conducta cotidiana en base al grado de participación físicamente activa y mentalmente consciente en la práctica religiosa y sin necesidad de esfuerzo físico o consciente en la conducta cotidiana (Stevens 2006: 21).

Después del Reino Nuevo, el desarrollo de cultos populares se vuelve más claro debido a la cantidad de evidencia disponible ${ }^{6}$ pero, en momentos previos, la falta

\footnotetext{
${ }^{6}$ Existe una extensa discusión sobre el momento de aparición y la
} 
de espacios delimitados y fácilmente reconocibles dificulta la identificación de materiales relacionados con la práctica religiosa, aunque no quiere decir que ésta no existiera (Baines 2001: 2). Sino que, algunas de las acciones religiosas implicadas en los cultos cotidianos eran llevadas a cabo en espacios apartados de los centros religiosos oficiales. Puesto que no todas estaban vinculadas con el culto estatal, la mayoría de las veces respondían a requerimientos particulares de las personas y estaban relacionadas con la existencia cotidiana o sus relaciones sociales (Baines 1991: 126). Esto supone la existencia de una gran diversidad de acciones religiosas, de las cuales la interacción con los dioses era solo un elemento más. En general, las cuestiones religiosas cotidianas también podían canalizarse hacia el ámbito de los muertos y ancestros (Stevens 2017).

En consecuencia, la religión popular en el antiguo Egipto se desarrolló en paralelo con la religión estatal y no existen motivos para creer que una y otra fueron contradictorias o excluyentes (Stevens 2006; Bickel 2003; Kemp 1995b). El período de Amarna presenta diferencias respecto de otros, dado que requería la intermediación de la realeza para la conexión con la divinidad. Sin embargo, tanto la elite como los egipcios comunes pudieron compartir ciertas prácticas religiosas no estatales y que se ocupaban de los problemas personales y cotidianos. Cuando la elite participaba en el culto oficial solo cubría una parte de sus necesidades y aspiraciones religiosas, mientras que todo lo relacionado con el sufrimiento y la pérdida, lo compartía con el resto de la sociedad (Baines 1987: 97; Bickel 2003: 66). Por ello, las deidades tradicionales pudieron ser objeto de devoción, incluso durante el período de Amarna, para las esferas más altas de la religión -a nivel estatal- así como de las medias y bajas -a nivel local y comunal-, al proporcionar alternativas válidas para la vida cotidiana (Baines 1987: 96).

Las fuentes documentales correspondientes a registros estatales raramente incluyen información sobre cultos populares, aunque pudieron ser comunes para todos (Baines 1991: 150). A lo que se suma una relativamente escasa evidencia sobre las prácticas personales y sus espacios de acción. El efecto omnipresente de la religiosidad individual (Bickel 2002: 66), que llevaba a un plano interno y casi continuo la relación de los egipcios con lo religioso ${ }^{7}$, también complica su estudio, debido a la inexistencia de registros (Baines 1991: 149). Sin embargo, el desafío de analizar una dinámica de las relaciones sociales, propias de la comunidad de Amarna, habilita analizar el alcance de ciertas prácticas religiosas a partir de la evidencia material.

expansión de prácticas religiosas personales (Assmann 2004; Baines 1987; Baines y Frood 2011; Bickel 2003; Kemp 1995b; Luiselli 2005, entre otros).

${ }^{7}$ El término religiosidad individual se utiliza de modo similar al de 'piedad personal' utilizado por autores como Breasted (1912) y Baines (1991) en referencia al contacto directo entre personas y deidades.

\section{Espacios}

El estudio de los espacios en el asentamiento Amarna supone comprender que las prácticas religiosas no se concentraban en un único lugar delimitado por el estado para tal fin, sino que se desarrollaban en múltiples áreas (Stevens 2006: 22), incluyendo así hogares, capillas y tumbas (Friedman 1994: 96). Los contextos en que las personas comunes visitaban los templos estatales tenían que ver, principalmente con festivales pero en Amarna estaban conectados con las apariciones de la familia real y se desarrollaban en torno a la vía procesional y los templos principales (Kemp 2012). En cambio, los espacios destinados a la realización de cultos populares fueron diversos, ya que las deidades se podían adorar en altares familiares, en capillas votivas privadas y en santuarios construidos y dirigidos por la comunidad local (Pinch 1993: 350).

Quizás por la falta de lugares físicos para construir espacios de culto privado en los hogares fue que una parte de las prácticas religiosas cotidianas se realizaban reutilizando lugares que, en otras ocasiones, fueran destinados para otras actividades. En este sentido, se interpreta la decoración de habitaciones con representaciones de las deidades Bes y Tawret en edificios de Deir el-Medina y Amarna (Pinch 1993: 126; Kemp 1995b: 30). Pudiendo vincularse esta adecuación con el traslado y reubicación de gente de la primera localización, como trabajadores en la segunda, que llevaran consigo sus deidades y prácticas. También se encontraron altares, ubicados frente a habitaciones que funcionaban como 'sala de estar', con espacios para colocar estatuas o estelas (Friedman 1994: 110). Así, se observa que el hábitat cotidiano no necesariamente tenía divisiones precisas por función, pues una misma habitación se utilizaba para diversas actividades, como el descanso, la convivencia o la práctica religiosa (Moeller 2015). En tanto, las áreas externas destinadas al culto, ubicadas en el Pueblo de Trabajadores, poseían altares ubicados en plataformas a las que se accedía por escaleras, en espacios amurallados, que pudieron estar parcial o completamente techados.

Según Spence (2015), era común alternar el uso de los espacios, ya que las tareas domésticas podían ser desarrolladas en el interior de los hogares, como también en áreas abiertas, como en los patios. Estas, lejos de ser unidades separadas y aisladas eran compartidas por la comunidad (Rocha da Silva 2018: 307). Por ello, es de esperar la creación de espacios destinados al culto tanto en el interior como en el exterior de los hogares conectando la vida doméstica con las actividades religiosas y funerarias en un uso comunitario (Bomann 1991).

En otras áreas residenciales, Kemp (1995b) señala la existencia de santuarios cercanos a los hogares de la elite. Uno de ellos contenía una estela dedicada a la tríada 
de Elefantina: Khnum, Anukis y Satis (Seidlmayer 1983: 204-206), además de una pequeña colección de vasijas de piedra, joyas esmaltadas, y una estatuilla que se asocia con la posible existencia de un pequeño santuario de Hathor en la Ciudad Principal (Kemp 1995b: 30; Borchardt y Ricke 1980: 111-112).

La distribución de santuarios en Amarna muestra dos niveles muy diferentes, ya que se los encuentra de forma excepcional en las principales áreas de la ciudad, mientras que en el Pueblo de Trabajadores son frecuentes, en donde aparece uno cada tres casas y ocupan un espacio aproximadamente igual a la mitad del pueblo. Esto nos permite inferir que los habitantes de Amarna habrían creado pequeños santuarios cuando las condiciones eran convenientes (Kemp 1995b: 32) y que existieron áreas de la ciudad más apropiadas que otras para la práctica religiosa en sus versiones cotidiana y comunitaria.

\section{Hallazgos}

Los hallazgos contemplados en este trabajo constan de joyería (aros y anillos), amuletos, moldes de arcilla, fragmentos de arquitectura, relieves, ostracas y estatuas, vasijas y objetos votivos, como pequeñas estatuillas. Cada uno de ellos será considerado en relación a su simbolismo y asociación con el tipo de culto que representan.

Los objetos de joyería -y sus moldes- fueron hallados en fragmentos $y$, en general, también pueden ser considerados sinónimo de amuletos ya que la mayoría de las joyas egipcias poseían ese valor (Pinch 1994: 106). Se asocian con representaciones o formas de jeroglíficos y tanto con representaciones de la religión popular como con la estatal.

Las prácticas religiosas tradicionales incluían el uso de amuletos tanto en enterramientos, para proteger al difunto en el Más Allá, como en la vida cotidiana (Andrews 1994: 6). Algunos se usaron temporalmente en situaciones de crisis, mientras que otros eran llevados regularmente para protección o beneficio permanente (Pinch 1994: 105-106). Su abundante pero dispersa presencia en diferentes localizaciones, implica que pudieron ser considerados necesarios tanto para la elite como para el resto de la sociedad.

En cuanto a las estatuas y restos de arquitectura, se relacionan generalmente con las producciones del estado, como los grandes templos, aunque en ocasiones también lo están con capillas y altares de uso privado con presencia de nichos y estelas. Las vasijas aparecen decoradas en asociación a todas las prácticas religiosas y habrían sido utilizadas como receptáculos durante rituales. Los ostracas, restos de cerámica utilizados para realizar inscripciones o representaciones a modo de borrador, aparecen asociados a las prácticas religiosas populares. Por último, las estatuillas y objetos votivos suelen estar en asociación con representaciones de la fertilidad y los cultos domésticos.

Las representaciones más abundantes dentro de los hallazgos considerados son los signos jeroglíficos $n f r$ y 'nh. El primero simbolizaba aspectos como la belleza, la bondad y el bienestar y estaba asociado con la fertilidad, mientras que el segundo era utilizado para escribir la palabra 'vida' y se llevaba como amuleto por la creencia de que su uso otorgaba o alargaba la vida. Su representación puede ligarse tanto a una funda porta falo como a una correa de sandalia (Pinch 1994: 110), ambos fueron símbolos del poder del faraón como creador y estabilizador del orden y, por tanto, dador de vida.

La palabra $\underline{d} d$ significa 'estabilidad' o 'inmutabilidad' y se encuentra, en general, representado en moldes y amuletos. Aunque al principio habría sido un pilar hecho de cañas, con el tiempo se asoció con la columna vertebral de Osiris (dios de los muertos). Vida, estabilidad y dominio (w3s), fueron las tres cualidades que los dioses tradicionalmente otorgaban a los faraones, de ahí su conexión con el orden y el bienestar (Pinch 2002: 128).

El signo $w \underline{d} 3 t$, 'ojo de Horus', se utilizaba en relación con Osiris y Horus (dios asociado a la realeza y con el poder del faraón). De acuerdo con el mito, Horus ofreció el ojo curado de su padre muerto (Osiris) y su poder fue lo que le devolvió la vida. Era llevado como amuleto ya que sus propiedades para la protección se relacionaban con su representación en la placa con la que se cubría la incisión del embalsamamiento de la momia. De esta manera, no solo prevenía de todas las influencias malignas, sino también que curaba mágicamente las heridas (Andrews 1994: 43).

Los objetos relacionados con la muerte, como los amuletos $w \underline{d}$ st y $d \underline{d}$, cobraron especial relevancia durante el período de Amarna, ya que uno de los efectos de la reforma fue eliminar la presencia a Osiris y suprimir los rituales funerarios alrededor de esta deidad (Martin 1989). Sin embargo, hay evidencia de hallazgos relacionados con aquel dios y de la continuidad en el uso de shabtis ('el que responde'), estatuillas consideras como los colaboradores del difunto en el Más Alla, las cuales eran asociadas al culto osiriaco (Smith 2017: 294).

En cuanto a los hallazgos relacionados directamente con deidades, los más abundantes son de Bes, Tawret y Hathor por su conexión con la fertilidad y los partos. Las deidades representadas bajo símbolos o a través de sus figuras moldeadas habrían sido usadas especialmente por mujeres y niños. Su producción no estaba patrocinada por el estado, con lo cual se explica la baja calidad de los mismos, además del hecho de que no estaban realizados bajo los cánones del estilo artístico oficial (Boyce 1995: 342). 
Bes se representaba con características de león (con melena y cola), a veces desnudo, con las patas arqueadas, largas plumas, las manos sobre sus caderas y, en ocasiones, tocando un tambor, dado que los ruidos ahuyentaban el mal. Era una de las deidades que asistía en el momento del parto (Andrews 1994: 39-40).

Tawret se representaba con forma de hipopótamo. La versión masculina era temida por los egipcios por su fuerza destructiva, mientras que la femenina era respetada como una ferviente protectora de sus crías, y la encarnación del poder vivificante del agua (Pinch 2002: 141-142).

Hathor fue la diosa que ayudaba a las mujeres a dar a luz, a los muertos a renacer y a la renovación del cosmos. Esta deidad podía ser la madre, consorte e hija del creador dios sol y era representada como una mujer usando un disco solar rojo entre un par de cuernos de vaca que salían de su cabeza, o directamente como una vaca. El nombre de Hathor significa 'dominio (o mansión) de Horus' y debió relacionarse con la idea de esta diosa como madre del dios de la realeza (Pinch 2002: 137).

Además de estas deidades, se podía acudir a la ayuda de los antepasados para solicitar, por ejemplo, nacimientos (Desroches Noblecourt 1953: 39), o que estos (los antepasados) intermediaran ante los grandes dioses. Las súplicas podían escribirse en estatuillas con la mujer desnuda, sosteniendo a un niño o cuerpos femeninos, con tatuajes o pinturas corporales que solían funcionar como objetos votivos (Pinch 1994: 126).

Dada la presencia de estos hallazgos en Amarna y comparándolos con el caso de Deir el-Medina, no parece que las creencias o prácticas asociadas con la religión popular fueran desaprobadas por el sacerdocio de los cultos estatales (Pinch 1993: 357). Además, la presencia de Bes y Tawret en escenas de nacimientos de la dinastía XVIII y en representaciones de palacios del Reino Nuevo, sugiere que las creencias populares relacionadas con la fertilidad se encontraban también vigentes en los niveles más altos de la sociedad egipcia. Para Pinch (1993: 359), la diosa Hathor podría verse como indicador de la continuidad de su devoción, atravesando a todos los niveles de la sociedad, ya que tenía una posición fundamental entre la religión popular y estatal.

Previo al período de Amarna existía una multiplicidad de dioses a los que apelar a través de ofrendas y oraciones (Teeter 2011: 194). Pero, pese a la imposición de Atón, la religión de Amarna no habría confrontado directamente con la religión tradicional, ya que esta, se centró en la figura del gobernante, instalando la idea de que el faraón era el único capaz de lograr indulgencias y favores divinos (Assmann 1980).

La nueva religión fue direccionada hacia unos pocos y tuvo un impacto restringido en las altas esferas de la elite. No contemplaba la idea de 'magia', que hasta ese momento estaba disponible para quien la necesitara, como fuerza efectiva en la constitución y manipulación del mundo. Sin embargo, amuletos, cartas y objetos votivos relacionados con creencias mágicas atonianas y tradicionales, fueron encontrados en todas las áreas del sitio Amarna. Esto parecería dar cuenta de que la elite aceptó las nuevas creencias, pero no abandonó la religión tradicional (Baines 1991: 189). Según Teeter (2011), dentro de todos los sistemas religiosos hay escépticos que hacen posible las desviaciones de la línea oficial. Como prueba de esta diversidad de creencias, amuletos y objetos votivos representando a los dioses Bes y Tawret, Hathor y representaciones del faraón y su esposa personificando a Shu y Tefnut ${ }^{8}$ han sido encontrados en distintos sectores de las excavaciones en Amarna.

La posibilidad de que las diversas prácticas religiosas en Amarna, relacionadas tanto con el culto estatal como con cultos populares y/o tradicionales, se desarrollaran no sólo en áreas específicamente delimitadas para el culto religioso y atravesaran a toda la población del sitio, tanto elite como no elite, requiere comprender el tipo y cantidad de evidencia distribuida.

\section{Culto estatal y culto popular en Amarna}

\section{Metodología y características de la muestra}

Se seleccionaron $\mathrm{N}=2535$ hallazgos conformando una muestra representativa a los fines de este trabajo. Su estudio busca conocer la tendencia cuantitativa de aspectos observados cualitativamente en la religión en Amarna con respecto a la existencia de una relación entre la cantidad y distribución de los materiales.

La muestra analizada consta de un conjunto de evidencia hallada en distintas áreas del sitio el-Amarna y que se encuentra publicada en diversas fuentes. La principal corresponde a la base de datos Small Finds data base, disponible en la página Amarna Project (2017). Esta contiene información detallada sobre $\mathrm{N}=7500$ pequeños hallazgos de la EES entre los años 1921 y 1936 y se confeccionó a partir de las publicaciones de City of Akhetaten I (Peet y Woolley 1923), II (Frankfort et al. 1933) y III (Pendlebury 1951). Para este trabajo se agregó a la muestra objetos relevados con anterioridad en las excavaciones efectuadas entre los años 1907 y 1914 por la Deutsche Orient-Gesellschaft (DOG), disponibles en la publicación del año 1980 de Borchardt et al. En base a los objetos publicados posteriormente en los informes periódicos de Barry Kemp, quien excava Amarna desde hace más de 40 años, se pudieron adicionar más hallazgos disponibles en los Amarna Reports, en la tesis

\footnotetext{
8 Asociado con la tríada divina conformada por Atón/Atum, Akhenatón/Shu y Nefertiti/Tefnut (Hornung 1999 [1971]: 158-159) muestra el desplazamiento de divinidades, pero la continuidad de la estructura religiosa tradicional.
} 
publicada de Anna Stevens, Private Religion at Amarna: The Material Evidence (Stevens 2006), en Busy Lives in the Main City (Kemp et al. 2010) y en Akhenaten's Workers: The Amarna Stone Village Survey (Stevens 2012).

Las categorías que se expondrán a continuación se ajustan con la hipótesis de trabajo que sostiene que Amarna fue un espacio en el que se desarrollaron diferentes prácticas religiosas y, mediante el análisis de los hallazgos relacionados con ellas, se puede acceder a la comprensión de su funcionamiento. Teniendo la particularidad de ser categorías estrictas, en cuanto a los requisitos que deben cumplir los hallazgos para ser incluidos (Ramenofsky y Steffen 1998), se privilegió la asociación directa entre deidades y/o temáticas, indicando que estas características se conectaron con algún tipo de práctica religiosa, pero sin considerar posibles derivaciones, que pudiesen involucrar mayor subjetividad.

Así, se construyeron las categorias a partir de las características y funciones de la evidencia que las componen (sensu Schiffer 1991: 14), es decir sus inscripciones, iconografía y formas, quedando constituidas de la siguiente manera: 1) Atón, 2) Realeza, 3) Amuletos jeroglíficos, 4) Amón, 5) Hathor, 6) Bes, 7) Tawret y 8) Fertilidad.

1) 'Atón' incluye joyería y restos de estatuas y arquitectura con referencias a este dios, sean inscripciones jeroglíficas o iconografía solar; 2) 'Realeza' contiene joyería, vasijas, ostracas y restos de arquitectura relacionados con el faraón, su esposa, sus hijas, sus antecesores y sucesores inmediatos, sean representaciones y/o inscripciones, además de uraeus por ser símbolo de la realeza; 3) 'Amuletos jeroglíficos' comprende materiales relacionados con joyería, y otros denominados 'amuletos' por la EES y contienen inscripciones de signos como el jeroglífico $w \underline{d} z t$ ('ojo de Horus'), $\underline{d} d$ ('estabilidad'), w3S ('cetro was'), 'nfr' ('belleza') y ''nh' ('vida'); 4) 'Amón' posee ostracas, estelas y joyería con alguna alusión a la deidad y en general está relacionado con nombres de la realeza; 5) 'Hathor' incluye vasijas, joyería y estatuillas con el nombre o la representación de la diosa, así como también sus formas de representación como vaca; 6) 'Bes' está compuesta por estatuillas, joyería y restos de arquitectura conectadas con la diosa; 7) 'Tawret' incluye estatuillas, joyería y restos de arquitectura relacionados con la deidad con el nombre o la representación de la diosa o representaciones de hipopótamos; y 8) 'Fertilidad' contiene representaciones de camas en miniaturas o partes de estas, además de figuras femeninas y de niños.

Las áreas de dónde proceden los elementos que conforman la muestra, fueron tomadas de la base de datos de la EES (Amarna Project 2017). Estando distribuidos entre: el Pueblo de Trabajadores, la Ciudad Central, la Ciudad Principal, el Marú Atón, la Ciudad Norte, el Suburbio Norte y el Palacio Norte.

A partir de las ocho categorías creadas, se ordenaron los hallazgos según su frecuencia de aparición en cada una de las áreas y se dispusieron en un gráfico de barras (Figura 2). Dado que cada categoría se relaciona con diversas prácticas religiosas, podemos inferir que su distribución da cuenta de los diferentes contextos de usos en que funcionaron los hallazgos, dando muestra de que cada categoría no reviste un uso exclusivo dentro de un segmento social de la población.

En las siguientes líneas, este acercamiento cualitativo será comparado con la prueba estadística de Chi-cuadrado (Chi2), mediante la cual se espera reconocer patrones de relación no visibles y las intensidades con que se encuentran asociadas categorías y áreas.

\section{Análisis estadístico de los datos y resultados}

Las pruebas estadísticas se utilizan para realizar inferencias acerca de ciertos aspectos de un conjunto de datos, proyectándose de lo específico (muestra) a lo general (población). Permiten medir la confiabilidad de la inducción en términos de probabilidad y así, para cada inferencia se puede identificar una determinada probabilidad para su aceptación, siendo esta la que determinará si la evidencia apoya la hipótesis propuesta, dado que, si esta probabilidad es superior al 0,050, se rechazará (Canavos 1998).

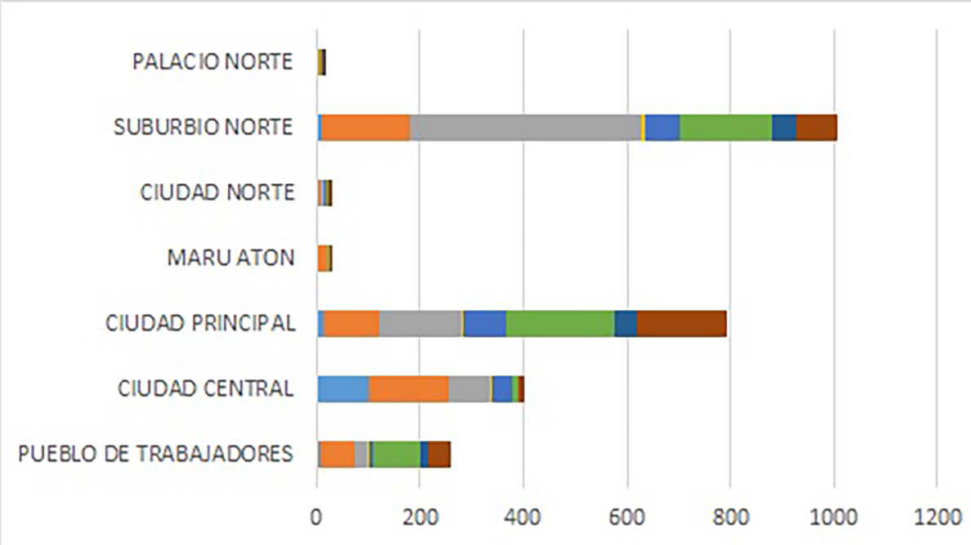

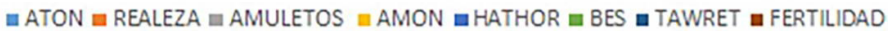

Un test adecuado para entender la relación entre dos variables cualitativas es el Chi2, ya que compara los valores empíricos con los calculados con respecto de una distribución teórica, cuya propuesta se sustenta a través de la formulación de la hipótesis nula (Shennan

Figura 2. Abundancia de $\mathrm{N}=2535$ hallazgos en categorías por áreas.

Figure 2. Abundance of $N=2535$ findings of categories by areas. 


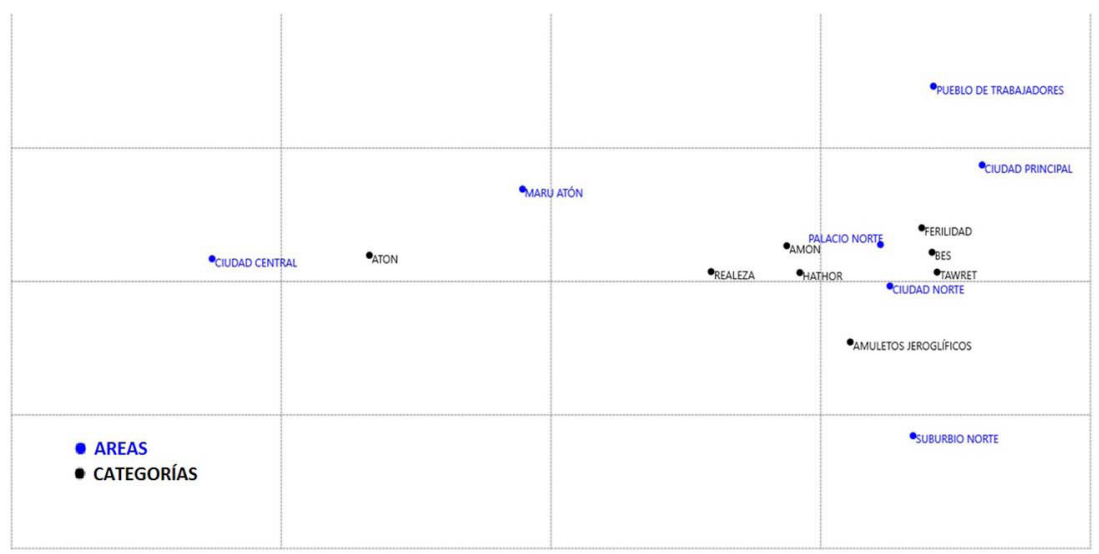

Figura 3. Correspondencia entre variables.

Figure 3. Correspondence between variables.

El valor arrojado fue de 0,0001, altamente significativo en relación al valor límite de 0,05 , por lo cual demostró, y confirmó, una baja probabilidad para aceptar la hipótesis nula de no asociación, y aceptar, en consecuencia la hipótesis alternatica de asociación.
1988). La hipótesis nula $-H_{0}-$, que se espera rechazar, sostendrá que la variabilidad de los datos puede atribuirse a procesos aleatorios; la hipótesis alternativa $-H_{1}-$, que se espera aceptar, dirá que efectivamente existe relación entre las variables analizadas. Si la prueba rechaza nuestra hipótesis nula, se concluirá que un factor causal propuesto influye en la variabilidad observada, pero si la probabilidad de ocurrencia supera el 0,050 (o el 5\%), la diferencia podría explicarse por azar y no en función de decisiones culturales.

Mediante el programa PAST, se aplicó el test de Chi2 a los datos para contrastar si los hallazgos asociados a la práctica religiosa se distribuyeron aleatoriamente en las diferentes áreas del sitio Amarna. Al valor de Chi2 de 949,68 , le corresponde una probabilidad baja $(8,7883 \mathrm{E}-$ 172) que no permite aceptar la hipótesis nula de no relación, indicando en cambio que la dependencia entre las filas (categorías) y columnas (áreas) era significativa (para un nivel de 0,05 y 42 grados de libertad). Pero, debido a que algunas de las categorías presentaron frecuencias muy bajas se consideró conveniente tomar el valor de probabilidad obtenido mediante el test de Monte Carlo para valorar mejor la relación puesta a prueba.

Este test, por ser más conservador que Chi2, difícilmente rechazará la hipótesis nula, ya que se basa en la comparación de las diferencias observadas en las tablas con las diferencias observadas en 10.000 repeticiones aleatorias de combinaciones semejantes (Barceló 2007).

\begin{tabular}{|l|c|c|c|c|}
\hline & $\begin{array}{c}\text { Cantidades de } \\
\text { hallazgos para } \\
\text { culto estatal }\end{array}$ & $\begin{array}{c}\text { Cantidades de } \\
\text { hallazgos para } \\
\text { culto no estatal }\end{array}$ & $\begin{array}{c}\text { Porcentajes } \\
\text { para culto } \\
\text { estatal }\end{array}$ & $\begin{array}{c}\text { Porcentajes } \\
\text { para culto no } \\
\text { estatal }\end{array}$ \\
\hline PT & 73 & 188 & $28 \%$ & $72 \%$ \\
\hline CC & 256 & 147 & $64 \%$ & $36 \%$ \\
\hline CP & 121 & 672 & $15 \%$ & $85 \%$ \\
\hline MA & 21 & 6 & $88 \%$ & $22 \%$ \\
\hline CN & 5 & 24 & $28 \%$ & $82 \%$ \\
\hline SN & 179 & 829 & $28 \%$ & $82 \%$ \\
\hline NP & 5 & 9 & $36 \%$ & $64 \%$ \\
\hline
\end{tabular}

La conclusión de las pruebas estadísticas es que las variables categoría y área están asociadas, por lo cual los hallazgos no se distribuyeron al azar. Una vez reconocido que existió alguna intencionalidad, se llevó a cabo un análisis interpretativo de los datos que permitió avanzar en la explicación de las intenciones de quienes utilizaron los materiales.

Mediante un gráfico de correspondencia, utilizado para investigar la magnitud y el tipo de relación entre filas y columnas de una tabla de contingencia, se convirtieron las diferencias entre los distintos valores de las variables área y categoría en distancias en el plano. A través de la aplicación de esta herramienta, se desprende que el área de la Ciudad Central está relacionada con las categorías 'Atón' y 'Realeza', mientras que esta misma área se mantiene alejada de las categorías 'Tawret', 'Bes' y 'Fertilidad'. Asimismo, todas las áreas, menos 'Ciudad Central', se encuentran a distancias relativamente similares de la categoría 'Fertilidad'. Por último, es de destacar que los hallazgos asociados a la esfera no estatal ('Amuletos jeroglíficos', 'Amón', 'Bes', 'Tawret', 'Hathor' y 'Fertilidad') se encuentran en las zonas residenciales, que habrían alojado una mayor cantidad de habitantes (Figura 3).

Mediante el análisis de los residuos ajustados, definido como la diferencia entre las frecuencias observadas y las esperadas, considerando el total de los datos, se evidencia la importancia que ciertas categorías tuvieron en el análisis. En el Pueblo de Trabajadores 'Bes' aparece más de lo esperado por azar, mientras que 'Amuletos jeroglíficos' aparece por debajo de lo esperado. La Ciudad Central, posee cantidades altas para

Tabla 1. Porcentajes de objetos de culto estatal vs. culto no estatal.

Table. 1. The percentages of state cult objects vs. non state cult objects. 

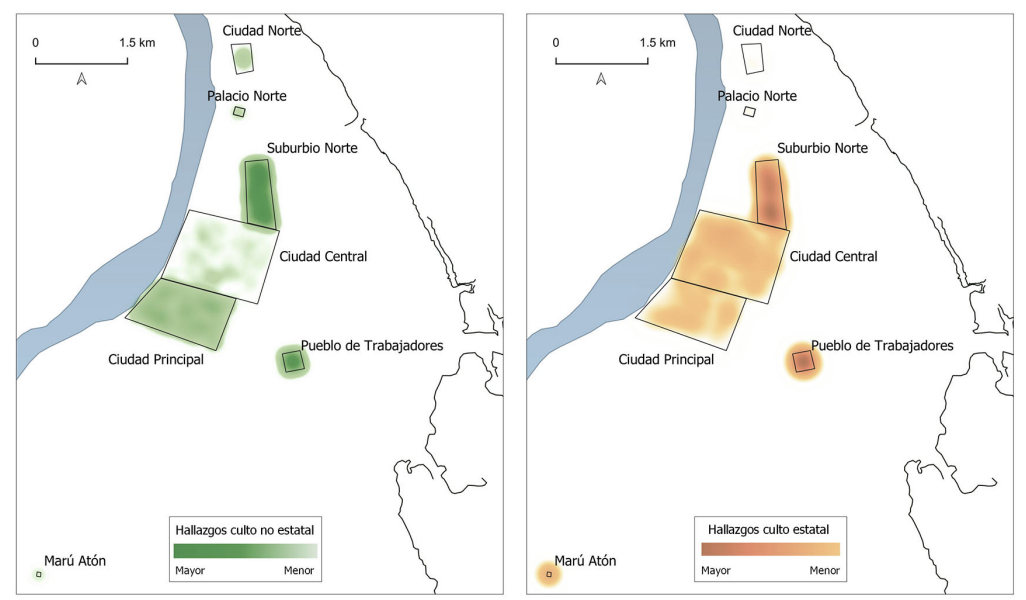

Figura 4. Mapas de calor con categorías de objetos de culto estatal y no estatal.

Figure 4. Color maps with categories of state cult objects and non state cult objects.

sociales y económicas, y donde coocurren evidencia estatal y no estatal.

Por último, se muestran las cantidades y porcentajes totales de las categorías en todo el sitio (Tabla 2). Los datos así presentados permiten entender el funcionamiento del culto estatal en paralelo con un culto popular, que

'Atón' y 'Realeza', mientras que 'Bes' aparece, junto con las categorías 'Hathor' y 'Amuletos jeroglíficos', con un valor menor de lo esperado. Asimismo, es de destacar los altos valores de 'Fertilidad', 'Hathor' y 'Bes' en el área de la Ciudad Principal y el de 'Amuletos jeroglíficos' para el Suburbio Norte.

Con el fin de mostrar una tendencia global, los datos se reagruparon en dos categorías más amplias: 'culto estatal' y 'culto no estatal'. La Tabla 1 ordena los hallazgos con el objetivo de visualizarlos en términos de porcentajes para cada una de las diferentes áreas. En la primera categoría ubicamos los asociados con el culto atoniano y la realeza mientras que en la segunda el resto de la evidencia, relacionada con el culto tradicional. La Ciudad Central posee un porcentaje alto para el culto estatal, mientras que lo contrario ocurre con la Ciudad Principal y el Suburbio Norte, ya que encontramos porcentajes altos para el culto no estatal. Resalta el caso del Pueblo de Trabajadores, donde el porcentaje de culto estatal es relativamente alto.

Los mismos datos se muestran mediante mapas de calor (Figura $4^{9}$ ) en los que se representaron los hallazgos a través de la densidad, indicadas con graduación de colores, hecho que nos permite detectar dónde se concentran las frecuencias. Si bien todas las categorías se distribuyen en todas las áreas del sitio, los materiales relacionados con el culto estatal (color naranja) lo hacen con mayor intensidad en el Suburbio Norte, en Marú Atón y en la Ciudad Central, sede de los templos de Atón y de edificios administrativos, mientras que su presencia se diluye hacia otras áreas. Por otro lado, los hallazgos relacionados con el culto no estatal (color verde) aparecen en tonos más intensos en el Suburbio Norte, el Pueblo de Trabajadores y la Ciudad Principal, todas áreas residenciales, donde habitaron todo tipo de jerarquías

\footnotetext{
${ }^{9}$ Creados con el programa Qgis con el fin de ilustrar la densidad de ubicación de la evidencia en las áreas en donde fueron registrados los hallazgos. Las posiciones asignadas a cada uno de los 2535 hallazgos que conforman la muestra son tentativas, dado que no disponemos de las coordenadas exactas de sus ubicaciones, sino sólo las referencias a unidades de registro.
} representa el $74 \%$ de los hallazgos relacionados con la práctica religiosa en Amarna. Es interesante señalar que su presencia indica que prácticas religiosas tradicionales se llevaron a cabo dentro de un sitio en el que, en términos oficiales, debía primar el culto al Atón y a la familia real, pero que, según este estudio, solamente representó un $26 \%$ del total de la evidencia material.

\section{Conclusiones}

La evidencia de prácticas religiosas aparece en todas las áreas de Amarna, con distintas intensidades e incluso donde no había espacios definidos para su desarrollo. Este hecho confirma que la religión era practicada en diferentes espacios, y no solo en el interior de los templos y en los lugares que ponían a las personas en presencia del faraón y la familia real. Ciertos objetos, como amuletos o figuras votivas, no se habrían utilizado en celebraciones religiosas estatales sino en rituales populares a nivel doméstico.

Las áreas de Amarna en las que encontramos mayor evidencia no estatal son las residenciales, lo cual se relacionaría con una relativa falta de control estatal, imposición del culto oficial o bien fueron los lugares en donde se encontraban intersticios entre lo público y lo privado, dando lugar a diversas prácticas. Áreas como el Palacio o la Ciudad Norte también poseen evidencia

Tabla 2. Cantidad y porcentajes de categorías.

Table 2. Quantity and percentages of categories.

\begin{tabular}{|c|c|c|}
\hline CATEGORÍA & CANTIDAD & PORCENTAJE \\
\hline ATON & 131 & 5 \\
\hline REALEZA & 529 & 21 \\
\hline AMULETOS & 728 & 29 \\
\hline AMON & 19 & 1 \\
\hline HATHOR & 200 & 8 \\
\hline BES & 498 & 20 \\
\hline TAWRET & 108 & 4 \\
\hline FERTILIDAD & 322 & 13 \\
\hline TOTALES & 2535 & 100 \\
\hline
\end{tabular}


de culto popular y, a su vez, habrían estado bastante afectadas por el estado, dada su proximidad con la residencia del faraón y la vía real.

En cuanto a la Ciudad Principal y el Suburbio Norte, poseen evidencia de culto popular, resultando el segundo de manera abundante. Ambas áreas estaban en cercanía con los principales templos de Atón y es difícil que las autoridades pudiesen ignorar la adoración de otras deidades. Una aparente falta de persecución en contra de otros dioses podría tomarse como una muestra más de que la adoración de las deidades tradicionales no estaba prohibida dentro de los límites de Amarna.

Por su parte, las áreas de la Ciudad Central y el Marú Atón poseen abunadante presencia de hallazgos relacionados con la religión oficial. En cuanto a la primera, alojaba templos, edificios administrativos y talleres destinados a la producción de objetos relacionados con Atón y la familia real. En tanto, la segunda, poseía un templo solar de auspicio estatal y estaba relacionada con la realeza.

A partir de lo presentado, podemos abonar la idea de que el culto al Atón se superpuso con la religión tradicional. Las categorías no estatales poseen una frecuencia de aparición bastante más alta de lo que podríamos esperar para la sede de un culto patrocinado por el estado. El análisis de los datos expuesto permite concluir que ambos, el estatal y el no estatal, convivieron durante el reinado de Akhenatón y esto siguió ocurriendo mientras Tutankhamón fue faraón. Su coexistencia solo fue posible porque abarcaba a la totalidad de la población, tanto elite como no elite formando parte del sistema de creencias vigente. A su vez, los cultos implicaron distintos momentos y lugares de relación con las divinidades que, no necesariamente eran contradictorios a los que se realizaban en otros momentos y lugares, en los cuales la relación con la divinidad estaba mediada, por ejemplo, por la familia real.

No obstante, existe la posibilidad de que la menor cantidad de hallazgos atonianos, en términos relativos, puede estar relacionada con el proceso mediante el cual se buscó eliminar los rastros de Akhenatón de la historia egipcia (damnatio memoriae), una vez restituido el culto a Amón. Otra posibilidad, está relacionada con el hecho de que este sitio continuara habitado luego de desaparecido el faraón, con la coexistencia de cultos.

Por último, lo expuesto invita a rever la reforma de Amarna en términos de largo plazo, tomando en consideración que fue un proceso con avances y retrocesos, iniciado años antes de la imposición de Atón por parte de Akhenatón y con consecuencias años después de su muerte. Así, es que no se puede hablar de un período de Amarna completamente excluyente de los cultos tradicionales siendo que la evidencia de estos cultos durante el gobierno de los sucesores de Akhenatón no representa necesariamente un final tajante para el atonismo, el culto a la familia real y las ideas impulsadas por la reforma.

Ciudad Autónoma de Buenos Aires, 6 de noviembre de 2019

\section{Agradecimientos}

Se agradece a quienes revisaron versiones previas del artículo, así como también a quienes lo evaluaron de forma anónima. Todas sus sugerencias contribuyeron a la mejora de este trabajo. También a los proyectos UBACyT (2018-2020, 20020170100688BA), 'Registros funerarios en el Cercano Oriente antiguo: análisis de estructuras, representaciones e inscripciones' y Proyecto PIP (20172019, 11220170100966CO), 'Arqueología de espacios funerarios: epigrafía y cultura material en el cercano oriente antiguo', CONICET, Buenos Aires, ambos dirigidos por la Dra, Liliana Manzi.

\section{Bibliografía}

Aldred, C. (1988). Akhenaten. King of Egypt. London: Thames \& Hudson.

Amarna, P. (2017). The Amarna Small Finds Database. https://www.amarnaproject.com/pages/recent_projects/ material_culture/small_finds/database.shtml

Andrews, C. (1994). Amulets of ancient Egypt. Austin: University of Texas Press.

Assmann, J. (1980). Die'Loyalistische Lehre'Echnatons. Studien zur altägyptischen Kultur, 8, 1-32.

Assmann, J. (1989). State and religion in the new kingdom. En W.K Simpson (Ed.), Religion and Philosophy in Ancient Egypt. Yale Egyptological Studies 3 (pp 55-88). New Haven: Yale University.

Assmann, J. (2004). Theological responses to Amarna. En G. Knoppers, y A. Hirsch (Eds.), Egypt, Israel, and the ancient Mediterranean world: Studies in honor of Donald B. Redford, Probleme der Ägyptologie 20 (pp. 179-191). Leiden and Boston: Brill.

Baines, J. (1987). Practical religion and piety. Journal of Egyptian Archaeology, 73(1), 79-98.

Baines, J. (1991). Society, morality and religious practice. En B. Schafer, J. Baines, L. Lesko y D. Silverman (Eds.), Religion in ancient Egypt (pp. 123-200). New York: Cornell University Press.

Baines, J. (2001). Egyptian letters of the New Kingdom as evidence for religious practice. Journal of Ancient Near Eastern Religions, 1(1), 1-31. 
Baines, J. y Frood, E. (2011). Piety, change and display in the New Kingdom. En M. Collier y S. Snape (Eds), Ramesside studies in honour of K.A. Kitchen (pp. 1-17). Bolton, Rutherford Press.

Barceló, J. (2007). Introducción al estudio de la variabilidad de las evidencias arqueológicas. Bellaterra: Universitat Autònoma de Barcelona.

Bickel, S. (2002). Aspects et fonctions de la déification d'Amenhotep III. Bulletin de I'Institut français d'archéologie orientale, 102, 63 - 90.

Bickel, S. (2003). "Ich spreche ständig zu Aton...": zur Mensch-Gott-Beziehung in der Amarna Religion. Journal of Ancient Near Eastern Religions, 3, 23-45.

Blyth, E. (2006). Karnak: evolution of a temple. New York: Routledge.

Bomann, A. (1991). The private chapel in ancient Egypt: A study of the chapels in the workmen's village at El Amarna with special reference to Deir el Medina and other sites. London: Kegan Paul International.

Borchardt, L., Ricke, H., y Ricke, H. (1980). Die Wohnhäuser in Teil el-Amarna (Ausgrabungen der Deutschen OrientGezellschaft in Teil el-Amarna V). Berlin: Mann

Boyce, A. (1995). Collar and necklace designs at Amarna: a preliminary study of faience pendants. En B. Kemp (Ed.), Amarna Reports VI (pp. 336-71). London: Egypt Exploration Foundation.

Breasted, J. (1912). Development of Religion and Thought in Ancient Egypt: Lectures delivered on the Morse Foundation at Union Theological Seminary. New York: Charles Scribner's Sons.

Canavos, G. (1998). Probabilidad y estadística. Aplicaciones y métodos. México: McGraw-Hill.

David, A. (2016). Akhenaten as the Early Morning Light: Revisiting the "Consecration" Ritual in Amarna. Journal of the American Research Center in Egypt, 52, 93-101.

Desroches Noblecourt, C. (1953) "Concubines du Mort" et Meres de Famille au Moyen Empire. Bulletin de I'Institut français d'archéologie orientale, 53, 7-48.

Frankfort, H., Fairman, H. W., y Pendlebury, J. D. S. (1933). The city of Akhenaten, Part 2, The north suburb and the desert altars: the excavations at Tell el Amarna during the season 1926-1932. London: Egypt Exploration Society.

Friedman, F. (1994). Aspects of domestic life and religion. En L. Lesko, L. (Ed.) Pharaoh's workers: the villagers of Deir el Medina (pp. 95-117). Chicago: Cornell University
Press.

Gabolde, M. (1998). D'Akhenaton à Toutânkhamon. Collection de I'Institut d'Archéologie et d'Histoire de I'Antiquité. Lyon: Université Lumière-Lyon 2, Institut d'archéologie et d'histoire de l'Antiquité

Gunn, B. (1916). The religion of the poor in ancient Egypt. Journal of Egyptian Archaeology 3(1), 81-94.

Hoffmeier, J. (2015). Akhenaten and the origins of monotheism. New York: Oxford University Press.

Hornung, E. (1999 (1971)). El Uno y los múltiples. Concepciones de la divinidad en el Egipto antiguo. Madrid: Trotta.

Hornung, E. (1999 (1995)). Akhenaten and the Religion of Light. Ithaca \& London: Cornell University Press.

Ikram, S. (1989). Domestic shrines and the cult of the royal family at el-'Amarna. Journal of Egyptian Archaeology, 75(1), 89-101.

Johnson, R. (1996). Amenhotep III and Amarna: Some New Considerations. Journal of Egyptian Archaeology, 8(82), 65-82.

Kemp, B. (1984). Patterns of activity at the Workmen's Village. En B. Kemp (Ed.), Amarna Reports I, (pp. 1-13). London: Egypt Exploration Foundation.

Kemp, B. (1987). The Amarna Workmen's Village in Retrospect. Journal of Egyptian Archaeology, 73, 21-50.

Kemp, B. (1992 (1989)). Antiguo Egipto. Anatomía de una civilización. Barcelona: Crítica.

Kemp, B. (1995a). Outlying Temples at Amarna. En B. Kemp (Ed.), Amarna Reports VI (pp. 411-425) London: Egypt Exploration Foundation.

Kemp, B. (2012). The city of Akhenaten and Nefertiti: Amarna and its people. London: Thames \& Hudson.

Kemp, B. (2016). How the City of Amarna came into being. The Akhenaten Sun, 22(2), 2-13.

Kemp, B., Stevens, A., y Buckland, P. (2010). Busy Lives at Amarna: Excavations in the Main City (grid 12 and the House of Ranefer, N49. 18). London: Egypt Exploration Society.

Kemp. B. (1995b). How Religious were the Ancient Egyptians? Cambridge Archaeological Journal, 5(1), 22-54

Luiselli, M. (2005). Personal Piety (modern theories related to). Los Angeles: UCLA Encyclopedia of Egyptology. Recuperado de http://escholarship.org/uc/item/49q0397q 
Manzi, L., Lemos, R y Pereyra, V (2015). O ritual como materialização de projetos políticos: uma comparação das representações rituais em tumbas de Amarna e Tebas. NEARCO - Revista Eletrônica de Antiguidade, 8(2), 111-140.

Mallinson, M. (1999). The Sacred Landscape. En R. Freed, J. Markowitz y S. H. D'Aurie (Eds.), Pharaohs of the Sun: Akhenaten, Nefertiti, Tutankhamun (pp. 72-79). Boston/ London: Museum of Fine Arts/ Thames \& Hudson

Martin, G.T. 1989. The Royal Tomb at El-'Amarna. London: Egypt Exploration Society.

Moeller, N. (2015). Multifunctionality and Hybrid Households: The Case of Ancient Egypt. En M. Müller (Ed.), Household Studies in Complex Societies: MicroArchaeological and Textual Approaches, OIS 10 (pp. 447-462). Chicago: Oriental Institute.

Murnane, W. y Meltzer, E. (1995). Texts from the Amarna Period in Egypt. Atlanta: Ga, Scholars Press.

Murnane, W. y van Siclen, C. (1993). The boundary stelae of Akhenaten. Studies in Egyptology. London: Kegan Paul International.

O' Connor, P. (1989). City and Palace in New Kingdom Egypt. Cahier de recherches de I'Institut de Papyrologie et d'Égyptologie de Lille, 11, 73-87.

Peet, T. y Woolley, C. (1923). The City of Akhenaten, I: Excavations of 1921 and 1922 at el-'Amarna. London: Egypt Exploration Society.

Pendlebury, J. (1951). The city of Akhenaten: Part III: The central city and the official quarters. The excavations at Tell-El-Amarna during the seasons 1926-1927 and 19311936. London: Egypt Exploration Society.

Petrie, W. (1894). Tell el Amarna. London: Methuen and Co.

Pinch, G. (1993). Votive Offerings to Hathor. Oxford: Griffith Institute.

Pinch, G. (1994). Magic in Ancient Egypt. London: British Museum Press.

Pinch, G. (2002). Handbook of Egyptian mythology. Santa Barbara/ California: ABC-CLIO.

Ramenofsky, A. y Steffen, A. (1998). Unit issues in archaeology: Measuring time, space, and material. Salt Lake City: University of Utah Press.

Redford, D. (1984). Akhenaten, the heretic king. Princeton: N.J., Princeton University Press.
Rocha da Silva, T. (2018). Reassessing Models in Gender and Domestic Space in New Kingdom Workmen's Villages. En S. L. Budin (Ed.), Gender and methodology in the ancient Near East: Approaches from assyriology and beyond (pp. 299-312). Barcelona: Edicions de la Universitat de Barcelona.

Schiffer, M. (1991). Formation processes of the archaeological record. Alburquerque: University of New Mexico Press.

Seidlmayer, S. (1983). Zu einigen Architekturinschriften aus Tell el-Amarna. Mitteilungen des Deutschen Archäologischen Instituts. Abteilung Kairo,39, 183-206.

Shaw, I. (1984). Ring bezels at el- Amarna. En B. Kemp (Ed.), Amarna reports I (pp. 124-132). London: Egypt Exploration Society.

Shennan, S. (1988). Quantifying archaeology. Great Britain: Edinburgh University Press.

Silverman, D., Wegner, J., y Wegner, J. (2006). Akhenaten and Tutankhamun: Revolution and restoration. Philadelphia: University of Pennsylvania Museum of Archaeology and Anthropology.

Spence, K. (2004). The Three-Dimensional Form of the Amarna House. Journal of Egyptian Archaeology, 90, 123-152.

Spence, K. (2010). Settlement Structure and Social Interaction at El-Amarna. En M. Bietak, E. Czerny y I. Forstner-Müller (Ed.), Cities and Urbanism in Egypt (pp. 289-298). Vienna: Österreichische Akademie der Wissenschaften.

Spence, K. (2015). Ancient Egyptian Houses and Households: Architecture, Artefacts, Conceptualization and Interpretation. En M. Muller (Ed.), Household Studies in Complex Societies. (Micro) Archaeological and Textual Approaches (pp. 83-99). Chicago: University of Chicago Press.

Stevens, A. (2017). Death and the City: The Cemeteries of Amarna in Their Urban Context. Cambridge Archaeological Journal 28(1), 103-126.

Stevens, A (2011). The Amarna Stone Village Survey and life on the urban periphery in New Kingdom Egypt. Journal of Field Archaeology, 36(2), 100-118.

Stevens, A. (2003). The material evidence for domestic religion at Amarna and preliminary remarks on its interpretation. Journal of Egyptian Archaeology, 89, 143-168.

Stevens, A. (2006). Private Religion at Amarna. The Material Evidence. Oxford: Archeopress. 
Stevens, A. (2012) Akhenaten's Workers: The Amarna Stone Village Survey. London: Egypt Exploration Society.

Stevens. A. (2015). The Archaeology of Amarna. Cambridge: Online publication. Recuperado de: https://www.oxfordhandbooks.com/view/10.1093/ oxfordhb/9780199935413.001.0001/oxfordhb9780199935413-e-31

Teeter, E. (1997). The presentation of Maat: Ritual and legitimacy in Ancient Egypt. Chicago/llinois: Oriental Institute of the University of Chicago.

Teeter, E. (2011). Religion and Ritual in Ancient Egypt. Cambridge: Cambridge University Press.
Williamson, J. (2017). Death and the Sun Temple: New Evidence for Private Mortuary Cults at Amarna. Journal of Egyptian Archaeology, 103(1), 117-123

Yomaha, S. (2004). La ceremonia de recompensa durante el reformismo amarniano: el ritual en la Ventana de Aparición. Tesis inédita de Licenciatura en Historia. Córdoba: Facultad de Filosofía y Humanidades, Universidad Nacional de Córdoba.

Yomaha, S. (2017). La topografía social en Amarna: los 'hombres nuevos' y la reconfiguración social tras la reforma. En Actas del XVI Jornadas Interescuelas/ Departamentos de Historia. Recuperado de: https://www. aacademica.org/000-019/4 